\title{
The Bruhat order on conjugation-invariant sets of involutions in the symmetric group
}

\author{
Mikael Hansson $]^{\dagger}$ \\ Department of Mathematics, Linköping University, Sweden
}

\begin{abstract}
Let $I_{n}$ be the set of involutions in the symmetric group $S_{n}$, and for $A \subseteq\{0,1, \ldots, n\}$, let$$
F_{n}^{A}=\left\{\sigma \in I_{n} \mid \sigma \text { has } a \text { fixed points for some } a \in A\right\} .
$$

We give a complete characterisation of the sets $A$ for which $F_{n}^{A}$, with the order induced by the Bruhat order on $S_{n}$, is a graded poset. In particular, we prove that $F_{n}^{\{1\}}$ (i.e., the set of involutions with exactly one fixed point) is graded, which settles a conjecture of Hultman in the affirmative. When $F_{n}^{A}$ is graded, we give its rank function. We also give a short new proof of the EL-shellability of $F_{n}^{\{0\}}$ (i.e., the set of fixed point-free involutions), which was recently proved by Can, Cherniavsky, and Twelbeck.
\end{abstract}

Résumé. Soit $I_{n}$ l'ensemble d'involutions dans le groupe symétrique $S_{n}$, et pour $A \subseteq\{0,1, \ldots, n\}$, soit

$$
F_{n}^{A}=\left\{\sigma \in I_{n} \mid \sigma \text { a } a \text { points fixes pour quelque } a \in A\right\} .
$$

Nous caractérisons tous les ensembles $A$ dont les $F_{n}^{A}$, avec l'ordre induit par l'ordre de Bruhat sur $S_{n}$, est un poset gradué. En particulier, nous démontrons que $F_{n}^{\{1\}}$ (c'est-à-dire, l'ensemble d'involutions avec précis en point fixe) est gradué, ce qui résout une conjecture d'Hultman à l'affirmative. Lorsque $F_{n}^{A}$ est gradué, nous donnons sa fonction de rang. En plus, nous donnons une nouvelle démonstration courte l'EL-shellability de $F_{n}^{\{0\}}$ (c'est-à-dire, l'ensemble d'involutions sans points fixes), établie récemment par Can, Cherniavsky et Twelbeck.

Keywords: Bruhat order, symmetric group, involution, conjugacy class, graded poset, EL-shellability

\section{Introduction}

Partially ordered by the Bruhat order, the symmetric group $S_{n}$ is a graded poset whose rank function is given by the number of inversions, and Edelman [4] proved that it is EL-shellable. Richardson and Springer [10] proved that the set $I_{n}$ of involutions in $S_{n}$ and the set $F_{n}^{0}$ of fixed point-free involutions are graded. Incitti [9] proved that the rank function of $I_{n}$ can be expressed as the average of the number of inversions and the number of exceedances, and that $I_{n}$ is EL-shellable. Hultman [8] studied (in a more general setting, which we shall describe shortly) $F_{n}^{0}$ and $F_{n}^{1}$, the set of involutions with exactly one

\footnotetext{
${ }^{\dagger}$ Email: mikael.hansson@liu.se

1365-8050 @ 2015 Discrete Mathematics and Theoretical Computer Science (DMTCS), Nancy, France
} 
fixed point. It follows that $F_{n}^{0}$ is graded and Hultman conjectured that the same is true for $F_{n}^{1}$. Can, Cherniavsky, and Twelbeck [3] recently proved that $F_{n}^{0}$ is EL-shellable.

We consider the following generalisation. For $a \in\{0,1, \ldots, n\}$, let $F_{n}^{a}$ be the conjugacy class in $S_{n}$ consisting of the involutions with $a$ fixed points, and for $A \subseteq\{0,1, \ldots, n\}$, let

$$
F_{n}^{A}=\bigcup_{a \in A} F_{n}^{a}
$$

Both $I_{n}$ and $F_{n}^{A}$ are regarded as posets with the order induced by the Bruhat order on $S_{n}$. Note that

$$
F_{n}^{A}=\left\{\sigma \in I_{n} \mid \sigma \text { has } a \text { fixed points for some } a \in A\right\} .
$$

Also note that for all elements in $I_{n}$, the number of fixed points is congruent to $n$ modulo 2 . Hence, we may assume that all members of $A$ have the same parity as $n$.

Depicted in Figures 1 and 2 , are the Hasse diagrams of $I_{4}, F_{4}^{0}$, and $F_{4}^{2}$.

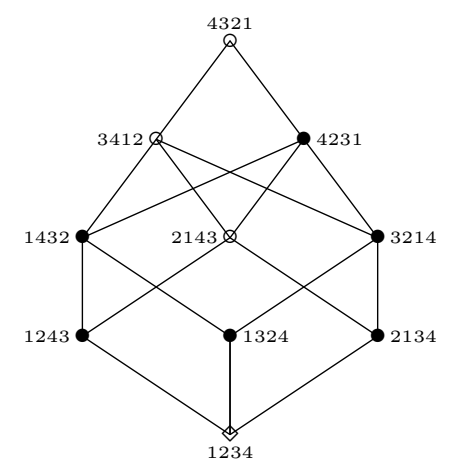

Figure 1: Hasse diagram of $I_{4}$ with the involutions with zero (०), two $(\bullet)$, and four $(\diamond)$ fixed points indicated.

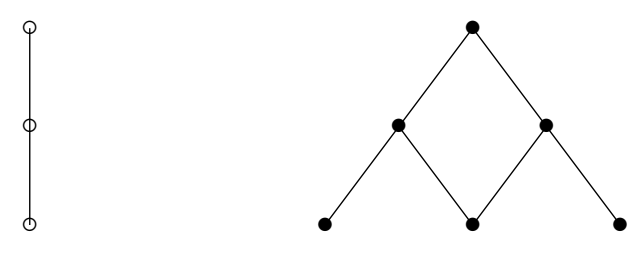

Figure 2: Hasse diagrams of $F_{4}^{0}$ (left) and $F_{4}^{2}$ (right).

Our main result is a complete characterisation of the sets $A$ for which $F_{n}^{A}$ is graded. In particular, we prove that $F_{n}^{1}$ is graded.

Informally, $F_{n}^{A}$ is graded precisely when $A-\{n\}$ is empty or an "interval," which may consist of a single element if it is 0,1 , or $n-2$. The following theorem, which is our main result, makes the above precise. It also gives the rank function of $F_{n}^{A}$ when it exists. 
Theorem 1 The poset $F_{n}^{A}$ is graded if and only if $A-\{n\}=\emptyset$ or $A-\{n\}=\left\{a_{1}, a_{1}+2, \ldots, a_{2}\right\}$ with $a_{1} \in\{0,1\}, a_{2}=n-2$, or $a_{2}-a_{1} \geq 2$. Furthermore, when $F_{n}^{A}$ is graded, its rank function $\rho$ is given by

$$
\rho(\sigma)=\frac{\operatorname{inv}(\sigma)+\operatorname{exc}(\sigma)-n+\tilde{a}}{2}+ \begin{cases}1 & \text { if } n \in A \\ 0 & \text { otherwise },\end{cases}
$$

where $\operatorname{inv}(\sigma)$ and $\operatorname{exc}(\sigma)$ denote the number of inversions and exceedances, respectively, of $\sigma$, and $\tilde{a}=$ $\max (A-\{n\})$. In particular, $F_{n}^{A}$ has rank

$$
\rho\left(F_{n}^{A}\right)=\frac{n^{2}-a^{2}-2 n+2 \tilde{a}}{4}+ \begin{cases}1 & \text { if } n \in A \\ 0 & \text { otherwise },\end{cases}
$$

where $a=\min A$.

The following result is direct consequence of Theorem 1

Corollary 2 The posets $F_{n}^{0}, F_{n}^{1}, F_{n}^{n-2}$, and $F_{n}^{n}$ are the only graded conjugacy classes of involutions in $S_{n}$. Furthermore, the rank function $\rho$ of $F_{n}^{0}$ and $F_{n}^{1}$ is given by

$$
\rho(\sigma)=\frac{\operatorname{inv}(\sigma)-\lfloor n / 2\rfloor}{2},
$$

and the rank function $\rho$ of $F_{n}^{n-2}$ is given by

$$
\rho(\sigma)=\frac{\operatorname{inv}(\sigma)-1}{2} .
$$

It is well known that $F_{n}^{n-2}$ is graded (in fact, it coincides with the root poset of the Weyl group $A_{n-1} \cong$ $S_{n}$ ). As was mentioned above, the gradedness of $F_{n}^{0}$ was proved by Richardson and Springer, and that of $F_{n}^{1}$ was conjectured by Hultman. These two posets are special cases of a more general construction from Hultman's paper [8], which we now describe (i)]

Given a finitely generated Coxeter system $(W, S)$ and an involutive automorphism $\theta$ of $(W, S)$ (i.e., a group automorphism $\theta$ of $W$ such that $\theta(S)=S$ and $\theta^{2}=$ id), let

$$
\iota(\theta)=\left\{\theta\left(w^{-1}\right) w \mid w \in W\right\}
$$

and

$$
\mathfrak{I}(\theta)=\left\{w \in W \mid \theta(w)=w^{-1}\right\}
$$

be the sets of twisted identities and twisted involutions, respectively. Clearly, $\iota(\theta) \subseteq \Im(\theta) \subseteq W$. Note that when $\theta=\mathrm{id}, \iota(\theta)$ and $\Im(\theta)$ reduce to the sets of the (ordinary) identity and (ordinary) involutions in $W$. Each subset of $W$ is regarded as a poset with the order induced by the Bruhat order on $W$. When $W$ is the symmetric group $S_{n}$, there is a unique non-trivial automorphism of $(W, S)$, mapping $s_{i}=(i, i+1)$ to $s_{n-i}$.

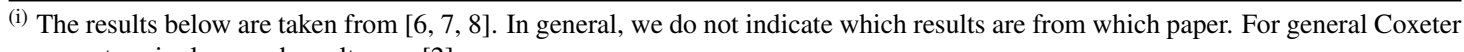
group terminology and results, see [2]. 
We say that $\theta$ has the no odd flip property if the order of $s \theta(s)$ is even or infinite for all $s \in S$ with $s \neq \theta(s)$. If $W$ is finite and irreducible, then $\theta$ has the no odd flip property, unless $W$ is of type $A_{2 n} \cong S_{2 n+1}$ or $I_{2}(2 n+1)$ for some $n \geq 1$, and $\theta$ is the unique non-trivial automorphism. The poset $\Im(\theta)$ is always graded. Furthermore, we have the following result, from which it follows that $F_{n}^{0}$ is graded, as we shall see.

Theorem A ([8, Theorem 4.6 and Proposition 6.7]) If $\theta$ has the no odd flip property, then $\iota(\theta)$ is graded with the same rank function as $\mathfrak{I}(\theta)$.

If $W$ is finite, it contains a greatest element $w_{0}$, and $\theta(w)=w_{0} w w_{0}$ defines an involutive automorphism of $(W, S)$. Since $\iota(\theta)=\left\{w_{0} w^{-1} w_{0} w \mid w \in W\right\}$ and $\mathfrak{I}(\theta)=\left\{w \in W \mid w_{0} w w_{0}=w^{-1}\right\}$,

$$
w_{0} \cdot \iota(\theta)=\left\{w^{-1} w_{0} w \mid w \in W\right\}
$$

and

$$
w_{0} \cdot \mathfrak{I}(\theta)=\left\{w_{0} w \mid w_{0} w w_{0}=w^{-1}\right\}=\left\{w_{0} w \mid\left(w_{0} w\right)^{2}=e\right\} .
$$

Since left (as well as right) multiplication by $w_{0}$ is a poset anti-automorphism (i.e., an order-reversing bijection whose inverse is order-reversing), $\iota(\theta)$ is isomorphic to the dual of $\left[w_{0}\right]$, where $\left[w_{0}\right]$ is the conjugacy class of $w_{0}$, and $\mathfrak{I}(\theta)$ is isomorphic to the dual of $I(W)$, where $I(W)$ is the set of involutions in $W$.

When $W$ is the symmetric group $S_{n}$, this $\theta$ is the unique non-trivial automorphism of $(W, S)$, and $I(W)=I_{n}$. For $n$ even, $\left[w_{0}\right]=F_{n}^{0}$, and for $n$ odd, $\left[w_{0}\right]=F_{n}^{1}$. Thus, it follows from Theorem A that $F_{n}^{0}$ is graded.

It was conjectured by Hultman [8, Conjecture 6.1] that $\iota(\theta)$ is graded when $W=A_{2 n}$. As we have seen, this is equivalent to $F_{n}^{1}$ being graded, which is the case (see Corollary 2). Since $\iota(\theta)$ is graded whenever $W$ is dihedral, as is easily seen, it therefore follows that $\iota(\theta)$ is graded whenever $W$ is finite and irreducible. From this, we get the following (we omit the proof):

Theorem 3 If $W$ is finite, then $\iota(\theta)$ is graded.

Let us also mention a connection to work by Richardson and Springer [10, 11], who studied a graded poset $V$ of orbits of certain symmetric varieties (depending on, inter alia, a group $G$ ). They did so by defining an order-preserving function $\varphi: V \rightarrow \Im(\theta) \subseteq W$ (where the Weyl group $W$ depends on, inter alia, $G$ ).

When $W=S_{n}, \Im(\theta)$ is the image of an injective $\varphi$ (for details, see [10, Example 10.2]). When $n$ is even, the same is true for $\iota(\theta)$ (see [10, Example 10.4] or [8, Example 3.1]). Thus, $I_{n}$ and $F_{n}^{0}$ are isomorphic to the duals of such posets $V$. Hence, $I_{n}$ and $F_{n}^{0}$ are graded.

However, these are not the only $F_{n}^{A}$ that occur as the image of a $\varphi$. To describe these sets, and for later purposes, define

$$
F_{n}^{\leq a}=\bigcup_{i \geq 0} F_{n}^{a-2 i} \quad \text { and } \quad F_{n}^{\geq a}=\bigcup_{i \geq 0} F_{n}^{a+2 i},
$$

and for $a_{2}=a_{1}+2 m$, where $m$ is a positive integer, let

$$
F_{n}^{a_{1}: a_{2}}=F_{n}^{\geq a_{1}} \cap F_{n}^{\leq a_{2}} .
$$


As described in [10], the image of $\varphi$ can be read off from the corresponding Satake diagram. It follows from Satake diagrams A III and A IV in Helgason [5, Table VI] that for each $a \leq n-2, F_{n}^{\geq a}$ is the image of a $\varphi$. (From Satake diagrams A I and A II, it follows that $\mathfrak{I}(\theta)$ and $\iota(\theta)$, respectively, are the images of such functions).

The remainder of this extended abstract is organised as follows. In Section 2 we agree on notation and gather the necessary definitions and previous results. Then, in Section 3 , we sketch the proof of our main result (Theorem 11. Finally, in Section 4 , we give a short new proof of the following result, which was recently proved by Can, Cherniavsky, and Twelbeck.

Theorem B ([3, Theorem 1]) The poset $F_{n}^{0}$ is EL-shellable.

\section{Notation and preliminaries}

Poset notation and terminology will follow [12]. In particular, if $P$ is a poset and $x \leq y$ in $P$, then $[x, y]=\{z \in P \mid x \leq z \leq y\}$ and $(x, y)=\{z \in P \mid x<z<y\}$. Furthermore, in a finite poset $P, \triangleleft$ denotes the covering relation, a chain $x_{0}<x_{1}<\cdots<x_{k}$ is saturated if $x_{i-1} \triangleleft x_{i}$ for all $i \in[k], P$ is bounded if it has a minimum (denoted by $\hat{0}$ ) and a maximum (denoted by $\hat{1}$ ), and $P$ is graded of rank $n$ if every maximal chain has length $n$. In this case, there is a unique rank function $\rho: P \rightarrow\{0,1, \ldots, n\}$ such that $\rho(x)=0$ if $x$ is a minimal element of $P$, and $\rho(y)=\rho(x)+1$ if $x \triangleleft y$ in $P$; $x$ has rank $i$ if $\rho(x)=i$. An $x$-y-chain is a saturated chain from $x$ to $y$.

Let $P$ be a finite, bounded, and graded poset. An edge-labelling of $P$ is a function $\lambda:\left\{(x, y) \in P^{2} \mid\right.$ $x \triangleleft y\} \rightarrow Q$, where $Q$ is a totally ordered set. If $\lambda$ is an edge-labelling of $P$ and $x_{0} \triangleleft x_{1} \triangleleft \cdots \triangleleft x_{k}$ is a saturated chain, let $\lambda\left(x_{0}, x_{1}, \ldots, x_{k}\right)=\left(\lambda\left(x_{0}, x_{1}\right), \lambda\left(x_{1}, x_{2}\right), \ldots, \lambda\left(x_{k-1}, x_{k}\right)\right)$. The chain is said to be increasing if $\lambda\left(x_{i-1}, x_{i}\right) \leq \lambda\left(x_{i}, x_{i+1}\right)$ for all $i \in[k-1]$, and decreasing if $\lambda\left(x_{i-1}, x_{i}\right)>\lambda\left(x_{i}, x_{i+1}\right)$ for all $i \in[k-1]$. An edge-labelling $\lambda$ of $P$ is an EL-labelling if, for all $x<y$ in $P$, there is exactly one increasing $x$-y-chain, say $x_{0} \triangleleft x_{1} \triangleleft \cdots \triangleleft x_{k}$, and this chain is lexicographically minimal, or lex-minimal, among the $x$-y-chains in $P$ (i.e., if $y_{0} \triangleleft y_{1} \triangleleft \cdots \triangleleft y_{k}$ is any other $x$-y-chain, then $\lambda\left(x_{j-1}, x_{j}\right)<\lambda\left(y_{j-1}, y_{j}\right)$, where $j=\min \left\{i \in[k] \mid \lambda\left(x_{i-1}, x_{i}\right) \neq \lambda\left(y_{i-1}, y_{i}\right)\right\}$; this is known as the lexicographic order). If $P$ has an EL-labelling, $P$ is said to be EL-shellable. The reason for this is the following result, due to Björner.

Theorem C ([1, Theorem 2.3]) Let $P$ be a finite, bounded, and graded poset. If $P$ is EL-shellable, then its order complex $\Delta(P)$ is shellable.

For $\sigma \in S_{n}$ and $(k, l) \in[n]^{2}$, let $\sigma[k, l]=|\{i \leq k \mid \sigma(i) \geq l\}|$. The Bruhat order on $S_{n}$ may be defined as follows (see, e.g., [2, Theorem 2.1.5]):

Definition 4 Let $\sigma, \tau \in S_{n}$. Then $\sigma \leq \tau$ if and only if $\sigma[k, l] \leq \tau[k, l]$ for all $(k, l) \in[n]^{2}$.

Let us turn to involutions in the symmetric group. Here, notation will follow [9].

Let $\sigma \in S_{n}$. A rise of $\sigma$ is a pair $(i, j) \in[n]^{2}$ such that $i<j$ and $\sigma(i)<\sigma(j)$. A rise $(i, j)$ is called free if there is no $i<k<j$ such that $\sigma(i)<\sigma(k)<\sigma(j)$. An inversion is a pair $(i, j) \in[n]^{2}$ such that $i<j$ and $\sigma(i)>\sigma(j)$. An element $i \in[n]$ is a fixed point of $\sigma$ if $\sigma(i)=i$, an exceedance if $\sigma(i)>i$, and a deficiency if $\sigma(i)<i$. Let $\operatorname{inv}(\sigma)$ and $\operatorname{exc}(\sigma)$ denote the number of inversions and exceedances, respectively, of $\sigma$. 
Let $\sigma \in I_{n}$. A free rise $(i, j)$ of $\sigma$ is suitable if it is an $f f$-rise (Type 1), an $f e$-rise (Type 2), an $e f$-rise (Type 3), a non-crossing ee-rise (Type 4), a crossing ee-rise (Type 5), or an $e d$-rise (Type 6). Here $f e$, e.g., means that $i$ is a fixed point of $\sigma$ while $j$ is an exceedance, and an ee-rise is crossing if $\sigma(i)<j$ and non-crossing otherwise.

The following definition is very important.

Definition 5 Let $\sigma \in I_{n}$ and let $(i, j)$ be a suitable rise of $\sigma$. We define a new involution $\mathrm{ct}_{(i, j)}(\sigma)$ as follows:

If $(i, j)$ is of Type 1 , then $\operatorname{ct}_{(i, j)}(\sigma)=\sigma(i, j)$.

If $(i, j)$ is of Type 2 , then $\mathrm{ct}_{(i, j)}(\sigma)=\sigma(i, j, \sigma(j))$.

If $(i, j)$ is of Type 3, then $\mathrm{ct}_{(i, j)}(\sigma)=\sigma(i, j, \sigma(i))$.

If $(i, j)$ is of Type 4, then $\operatorname{ct}_{(i, j)}(\sigma)=\sigma(i, j)(\sigma(i), \sigma(j))$.

If $(i, j)$ is of Type 5, then $\operatorname{ct}_{(i, j)}(\sigma)=\sigma(i, j, \sigma(j), \sigma(i))$.

If $(i, j)$ is of Type 6 , then $\operatorname{ct}_{(i, j)}(\sigma)=\sigma(i, j)(\sigma(i), \sigma(j))$.

See [9] Table 1] for pictures describing the action of $\mathrm{ct}_{(i, j)}$ on the diagram of $\sigma$.

Incitti characterised the covering relation in $I_{n}$ as follows.

Lemma 6 ([9, Theorem 5.1]) Let $\sigma, \tau \in I_{n}$. Then $\sigma \triangleleft \tau$ in $I_{n}$ if and only if $\tau=\operatorname{ct}_{(i, j)}(\sigma)$ for some (necessarily unique) suitable rise $(i, j)$ of $\sigma$.

If $\tau=\operatorname{ct}_{(i, j)}(\sigma)$ for some suitable rise $(i, j)$ of $\sigma$, let $\lambda(\sigma, \tau)=(i, j)$. By Lemma 6, this defines an edge-labelling of $I_{n}$ (with $\left\{(i, j) \in[n]^{2} \mid i<j\right\}$ totally ordered by the lexicographic order, i.e., $\left(i_{1}, j_{1}\right)<\left(i_{2}, j_{2}\right)$ if and only if $i_{1}<i_{2}$, or $i_{1}=i_{2}$ and $\left.j_{1}<j_{2}\right)$. Whenever we consider an edge-labelling of $I_{n}$, it is this one. If $\lambda(\sigma, \tau)=(i, j)$, then $(i, j)$ is the label on the cover $\sigma \triangleleft \tau ;(i, j)$ is a label on a chain if it is the label on some cover of the chain.

Let $\tau \in I_{n}$ and let $(i, j)$ be an inversion of $\tau$. If $(i, j)$ is a suitable rise of some $\sigma \in I_{n}$ and $\operatorname{ct}_{(i, j)}(\sigma)=$ $\tau$, then $\sigma$ is unique, and we write $\sigma=\operatorname{ict}_{(i, j)}(\tau)$.

For $\sigma<\tau$ in $I_{n}$, let $\operatorname{di}(\sigma, \tau)=\min \{i \in[n] \mid \sigma(i) \neq \tau(i)\}$.

We shall need the following results, due to Incitti:

Lemma 7 ([9, Theorem 5.2]) The poset $I_{n}$ is graded with rank function $\rho$ given by

$$
\rho(\sigma)=\frac{\operatorname{inv}(\sigma)+\operatorname{exc}(\sigma)}{2}
$$

Lemma 8 ([9. Theorem 6.2]) Let $\sigma<\tau$ in $I_{n}$. Then there is exactly one increasing $\sigma$ - $\tau$-chain, and it is lex-minimal.

Lemma 9 ([9, Theorem 7.3]) Let $\sigma<\tau$ in $I_{n}$. Then there is exactly one decreasing $\sigma$ - $\tau$-chain.

Remark 1 Since $\operatorname{ct}_{(i, j)}(\sigma)(i)>\operatorname{ct}_{(i, j)}(\sigma)(j)$, there is also exactly one "weakly" decreasing $\sigma$ - $\tau$-chain. This fact is used in Section 4 


\section{Sketch of the proof of the main result}

In this section, we prove, sketch the proofs of, or simply state, a number of lemmas and propositions, from which Theorem 1 easily follows.

The strategy for proving that a poset $F_{n}^{A}$ is graded is as follows. We first prove that $F_{n}^{A}$ has a maximum and that all its minimal elements have the same rank in $I_{n}$ (see Propositions 11 and 12). We then prove that if $\sigma, \tau \in F_{n}^{A}$, then $\sigma \triangleleft \tau$ in $F_{n}^{A}$ if and only if $\sigma \triangleleft \tau$ in $I_{n}$ (one implication is obvious). This is done in Lemmas 15,16 and 17 . Since $I_{n}$ is graded, it thus follows that $F_{n}^{A}$ is graded.

In particular, when $F_{n}^{A} \in\left\{F_{n}^{\leq a}, F_{n}^{\geq a}\right\}$, to prove that $\sigma \triangleleft \tau$ in $I_{n}$ if $\sigma \triangleleft \tau$ in $F_{n}^{A}$, we assume that $\sigma \Varangle \tau$ in $I_{n}$, and consider the increasing and the decreasing $\sigma$ - $\tau$-chains in $I_{n}$. We then prove that either the element in the increasing chain that covers $\sigma$, or the element in the decreasing chain that is covered by $\tau$, has to belong to $F_{n}^{A}$. This contradicts the fact that $\sigma \triangleleft \tau$ in $F_{n}^{A}$.

To prove that a poset $F_{n}^{A}$ is not graded, we consider an interval $[\sigma, \tau]$, and then construct two $\sigma$ - $\tau$-chains in $F_{n}^{A}$ of different lengths (see Propositions 19 and 20.

Let us first note the following fact:

Lemma 10 For all $n$ and all $A, F_{n}^{A}$ is graded if and only if $F_{n}^{A-\{n\}}$ is graded.

Proof: This is obvious if $n \notin A$. Otherwise, deleting the identity permutation gives a bijection between maximal chains in $F_{n}^{A}$ of length $k$ and maximal chains in $F_{n}^{A-\{n\}}$ of length $k-1$.

In the next two results, we describe the maximal and minimal elements of $F_{n}^{A}$. The proofs are not given here.

Proposition 11 For all $n$ and all $A, F_{n}^{A}$ has a $\hat{1}$. Furthermore, $\operatorname{inv}(\hat{1})=\frac{n-a}{2}(n+a-1)$ and $\operatorname{exc}(\hat{1})=$ $\frac{n-a}{2}$, where $a=\min A$.

Proposition 12 For all $n$ and all $A$, all minimal elements of $F_{n}^{A}$ have rank $(n-\max A) / 2$ in $I_{n}$.

Recall that

$$
F_{n}^{\leq a}=\bigcup_{i \geq 0} F_{n}^{a-2 i}, \quad F_{n}^{\geq a}=\bigcup_{i \geq 0} F_{n}^{a+2 i}, \quad \text { and } \quad F_{n}^{a_{1}: a_{2}}=F_{n}^{\geq a_{1}} \cap F_{n}^{\leq a_{2}},
$$

where $a_{2}=a_{1}+2 m$ for some positive integer $m$. Note that $F_{n}^{a_{1}: a_{2}}$ is not defined for $a_{1}=a_{2}$.

The following lemma will eventually allow us to conclude that $F_{n}^{\leq a}, F_{n}^{\geq a}$, and $F_{n}^{a_{1}: a_{2}}$ are graded.

Lemma 13 If every cover in $F_{n}^{A}$ is a cover in $I_{n}$, then $F_{n}^{A}$ is graded.

Proof: This follows from Lemma 7 and Propositions 11 and 12

The next lemma is used in the proofs of Lemmas 15, 16, and 17, which, together with Lemma 13, show that $F_{n}^{\leq a}, F_{n}^{\geq a}$, and $F_{n}^{a_{1}: a_{2}}$ are graded.

Lemma 14 Let $\sigma<\tau$ in $I_{n}$. Then the label $(i, j)$ on any cover in $[\sigma, \tau]$ satisfies $i \geq \operatorname{di}(\sigma, \tau)$.

Proof: Suppose $i<\operatorname{di}(\sigma, \tau)$ for the label $(i, j)$ on $\sigma \triangleleft \pi \leq \tau$. Then $\pi(k)=\tau(k)$ for $k<i$ and $\sigma(i)=\tau(i)$. However, it follows from Definition 5 that $\pi(i)>\sigma(i)$. Hence, $\pi[i, \sigma(i)+1]>\tau[i, \sigma(i)+1]$. By Definition 4, this contradicts the fact that $\pi \leq \tau$. Thus $i \geq \operatorname{di}(\sigma, \tau)$. The result follows by induction. 
Lemma 15 Let $\sigma \triangleleft \tau$ in $F_{n}^{\leq a}$. Then $\sigma \triangleleft \tau$ in $I_{n}$.

Proof: Assume that $\sigma \not \tau$ in $I_{n}$, and let $C_{I}=\sigma \triangleleft \sigma_{1} \triangleleft \cdots \triangleleft \sigma_{k} \triangleleft \tau$ be the increasing $\sigma$ - $\tau$-chain in $I_{n}$ and $C_{D}=\sigma \triangleleft \tau_{k} \triangleleft \cdots \triangleleft \tau_{1} \triangleleft \tau$ the decreasing $\sigma$ - $\tau$-chain in $I_{n}$. Since $\sigma \triangleleft \tau$ in $F_{n}^{\leq a}$, neither $\sigma_{1}$ nor $\tau_{1}$ belongs to $F_{n}^{\leq a}$.

Let $h=\operatorname{di}(\sigma, \tau)$, and let $\left(i_{\sigma}, j_{\sigma}\right)$ and $\left(i_{\tau}, j_{\tau}\right)$ be the labels on $\sigma \triangleleft \sigma_{1}$ and $\tau_{1} \triangleleft \tau$, respectively. Since $\sigma(h) \neq \tau(h)$, it follows from Lemma 14 that $h$ is in some label on $C_{I}$ and some label on $C_{D}$. Since $C_{I}$ is increasing, $i_{\sigma}=h$, and since $\sigma_{1} \notin F_{n}^{\leq a}, h$ is an exceedance of $\sigma$ (Type 5). Since $C_{D}$ is decreasing, $i_{\tau}=h$, and since $\tau_{1} \notin F_{n}^{\leq a}, h$ is a fixed point of $\tau_{1}$ (Type 1). Hence, $\sigma[h, h+1]>\tau_{1}[h, h+1]$. By Definition 4 , this contradicts the fact that $\sigma \leq \tau_{1}$.

Lemma 16 Let $\sigma \triangleleft \tau$ in $F_{n}^{\geq a}$. Then $\sigma \triangleleft \tau$ in $I_{n}$.

Proof: Assume that $\sigma \Varangle \tau$ in $I_{n}$, and let $C_{I}=\sigma \triangleleft \sigma_{1} \triangleleft \cdots \triangleleft \sigma_{k} \triangleleft \tau$ be the increasing $\sigma$ - $\tau$-chain in $I_{n}$ and $C_{D}=\sigma \triangleleft \tau_{k} \triangleleft \cdots \triangleleft \tau_{1} \triangleleft \tau$ the decreasing $\sigma-\tau$-chain in $I_{n}$. Since $\sigma \triangleleft \tau$ in $F_{n}^{\geq a}$, neither $\sigma_{1}$ nor $\tau_{1}$ belongs to $F_{n}^{\geq a}$.

Let $h=\operatorname{di}(\sigma, \tau)$, and let $\left(i_{\sigma}, j_{\sigma}\right)$ and $\left(i_{\tau}, j_{\tau}\right)$ be the labels on $\sigma \triangleleft \sigma_{1}$ and $\tau_{1} \triangleleft \tau$, respectively. Since $\sigma(h) \neq \tau(h)$, it follows from Lemma 14 that $h$ is in some label on $C_{I}$ and some label on $C_{D}$. Since $C_{I}$ is increasing, $i_{\sigma}=h$, and since $\sigma_{1} \notin F_{n}^{z a}, h$ is a fixed point of $\sigma$ (Type 1). Since $C_{D}$ is decreasing, $i_{\tau}=h$, and since $\tau_{1} \notin F_{n}^{\geq a}, h$ is an exceedance of $\tau_{1}$ (Type 5).

Let $m$ be such that $h$ is an exceedance of $\tau_{1}, \ldots, \tau_{m-1}$ and a fixed point of $\tau_{m}$ (with $\tau_{k+1}=\sigma$ ). Then the labels on $\tau \triangleright \tau_{1} \triangleright \cdots \triangleright \tau_{m}$ are $\left(h, j_{1}\right), \ldots,\left(h, j_{m}\right)$, where $j_{1}<j_{2}<\cdots<j_{m}$. Since $\tau_{1}>$ $\tau_{2}>\cdots>\tau_{m-1}, \tau_{1}(h)>\tau_{2}(h)>\cdots>\tau_{m-1}(h)$. Since $h$ is a fixed point of $\tau_{m}$ but an exceedance of $\tau_{m-1}$, the cover $\tau_{m} \triangleleft \tau_{m-1}$ is of Type 1 or 2 , whence $\tau_{m-1}(h)=j_{m}$ or $\tau_{m-1}(h)=\tau_{m}\left(j_{m}\right)>j_{m}$, respectively; hence, $\tau_{m-1}(h) \geq j_{m}$. Therefore, $j_{1}<j_{m} \leq \tau_{m-1}(h) \leq \tau_{1}(h)$. However, since the cover $\tau_{1} \triangleleft \tau$ is of Type $5, \tau_{1}(h)<j_{1}$, which is a contradiction.

Lemma 17 Let $\sigma \triangleleft \tau$ in $F_{n}^{a_{1}: a_{2}}$. Then $\sigma \triangleleft \tau$ in $I_{n}$.

The proof, which is omitted here, is largely a combination of the proofs of Lemmas 15 and 16

Proposition 18 The posets $F_{n}^{\leq a}, F_{n}^{\geq a}$, and $F_{n}^{a_{1}: a_{2}}$ are graded.

Proof: This follows from Lemmas 13, 15, 16 , and 17 .

In the following two results, we consider the sets $A$ for which $F_{n}^{A}$ is not graded.

Proposition 19 If there is an $i \in[2, n-4]$ such that $i \in A$ but $i-2, i+2 \notin A$, then $F_{n}^{A}$ is not graded.

The proof, which is not given here, is similar to, but easier than, the proof of Proposition 20

Proposition 20 If there is an $i \notin A$ and a positive integer $m$ such that $i-2, i+2 m \in A-\{n\}$, then $F_{n}^{A}$ is not graded.

Proof sketch: We first prove that $F_{k}^{\{0, k-2\}}$, where $k \geq 6$ is even, is not graded. Let $\sigma=12 \cdots(k-$ $2) k(k-1)$ and $\tau=k 23 \cdots(k-1) 1$, and consider the interval $[\sigma, \tau]$. We obtain a $\sigma$ - $\tau$-chain $C$ in $I_{k}$ by $k-2 f e$-rises with labels $(k-2, k-1),(k-3, k-2), \ldots,(1,2)$ (from $\sigma$ to $\tau$ ). We also obtain a 
$\sigma$ - $\tau$-chain in $I_{n}$ by $(k-2) / 2 f f$-rises with labels $(1,2),(3,4), \ldots,(k-3, k-2)$, followed by $(k-2) / 2$ crossing ee-rises with labels $(k-3, k-1),(k-5, k-3), \ldots,(1,3)$.

Let $\pi$ be the fixed point-free involution obtained after the $f f$-rises. Since each $f f$-rise decreases the number of fixed points and $I_{k}$ is graded, $(\sigma, \pi) \cap F_{k}^{\{0, k-2\}}=\emptyset$, and since each crossing ee-rise increases the number of fixed points and $I_{k}$ is graded, $(\pi, \tau) \cap F_{k}^{\{0, k-2\}}=\emptyset$. Hence, $C$ is a $\sigma$ - $\tau$-chain in $F_{k}^{\{0, k-2\}}$ of length $k-2$, while $\sigma \triangleleft \pi \triangleleft \tau$ is a $\sigma$ - $\tau$-chain in $F_{k}^{\{0, k-2\}}$ of length 2 . Thus $F_{k}^{\{0, k-2\}}$ is not graded. Figure 3 illustrates the situation when $k=6$.

Now we have to obtain the right number of fixed points. The details are not given here.

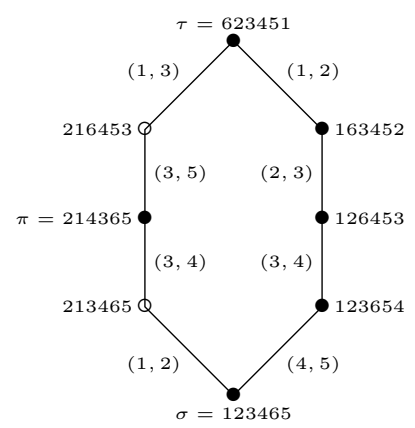

Figure 3: Two $\sigma$ - $\tau$-chains in $I_{6}$ of length 4 , and two $\sigma$ - $\tau$-chains in $F_{6}^{\{0,4\}}$ of length 4 (right) and length 2 (left); the involutions marked by a $\bullet$ belong to $F_{6}^{\{0,4\}}$, and the involutions marked by a $\circ$ belong to $I_{6}-F_{6}^{\{0,4\}}$. On the edges (covers in $I_{6}$ ) are the labels $(i, j)$.

We are now ready to prove our main result:

Proof of Theorem 1: The first claim follows from Lemma 10 and Propositions 18, 19, and 20, (It is readily checked that if $F_{n}^{A-\{n\}}$ does not belong to $\left\{\emptyset, F_{n}^{\leq a}, F_{n}^{\geq a}, F_{n}^{a_{1}: a_{2}}\right\}$, then either there is an $i \in[2, n-4]$ such that $i \in A$ but $i-2, i+2 \notin A$, or there are an $i \notin A$ and a positive integer $m$ such that $i-2, i+2 m \in A-\{n\}$.) The second claim follows from Lemma 7. Proposition 12 and Lemmas 15 , [16, and 17. The third claim follows from the second claim and Proposition 11

\section{EL-shellability of $F_{n}^{0}$}

In this section, we give a new proof of Theorem B, due to Can, Cherniavsky, and Twelbeck [3]. Our proof is largely based on the same main idea as their proof, together with the technique used in the proof of Lemma 15] The proof in [3] goes as follows:

Let $\sigma<\tau$ in $F_{n}^{0}$. It follows from, e.g., Theorem A and the paragraphs following it, that there exists a $\sigma$ - $\tau$-chain in $I_{n}$ that is contained in $F_{n}^{0}$. Let $C$ be the lex-maximal such chain. The idea of the proof is to show that $C$ is decreasing. Then, by reversing the lexicographic order on the set $\left\{(i, j) \in[n]^{2} \mid i<j\right\}$ (i.e., by letting $\left(i_{1}, j_{1}\right)<\left(i_{2}, j_{2}\right)$ if and only if $i_{1}>i_{2}$, or $i_{1}=i_{2}$ and $j_{1}>j_{2}$ ), one obtains an edgelabelling of $F_{n}^{0}$ such that in each interval, there is an increasing $\sigma$ - $\tau$-chain which is lex-minimal. By Lemma 9 and the remark following it, this is an EL-labelling of $F_{n}^{0}$. 
We use the same main idea, namely, to show that the decreasing $\sigma$ - $\tau$-chain in $I_{n}$ is contained in $F_{n}^{0}$, and then reverse the lexicographic order. However, we give a direct proof of this fact. By using the same technique as in the proof of Lemma 15 , we get a very short argument.

Lemma 21 Let $\sigma<\tau$ in $F_{n}^{0}$ and let $C_{D}=\sigma \triangleleft \tau_{k} \triangleleft \cdots \triangleleft \tau_{1} \triangleleft \tau$ be the decreasing $\sigma$ - $\tau$-chain in $I_{n}$, where $k \geq 1$. Then $\tau_{1}, \ldots, \tau_{k} \in F_{n}^{0}$.

Proof: Since the decreasing $\sigma$ - $\tau_{1}$-chain in $I_{n}$ is $\sigma \triangleleft \tau_{k} \triangleleft \cdots \triangleleft \tau_{2} \triangleleft \tau_{1}$, it suffices to prove that $\tau_{1} \in F_{n}^{0}$.

Let $h=\operatorname{di}(\sigma, \tau)$, let $C_{I}=\sigma \triangleleft \sigma_{1} \triangleleft \cdots \triangleleft \sigma_{k} \triangleleft \tau$ be the increasing $\sigma$ - $\tau$-chain in $I_{n}$, and let $\left(i_{\sigma}, j_{\sigma}\right)$ and $\left(i_{\tau}, j_{\tau}\right)$ be the labels on $\sigma \triangleleft \sigma_{1}$ and $\tau_{1} \triangleleft \tau$, respectively. Since $\sigma(h) \neq \tau(h)$, it follows from Lemma 14 that $h$ is in some label on $C_{I}$ and some label on $C_{D}$. Since $C_{I}$ is increasing, $i_{\sigma}=h$, and since $\sigma$ has no fixed points, $h$ is an exceedance of $\sigma$ (Type 4, 5, or 6). Since $C_{D}$ is decreasing, $i_{\tau}=h$, and were $\tau_{1} \notin F_{n}^{0}, h$ would be a fixed point of $\tau_{1}$ (Type 1). Hence, by Definition $4, \tau_{1} \in F_{n}^{0}$.

We can now complete the proof of Theorem B.

Proof of Theorem B: Let $\sigma<\tau$ in $F_{n}^{0}$. By Lemma 21 the decreasing $\sigma$ - $\tau$-chain in $I_{n}$ is contained in $F_{n}^{0}$. If we can show that this chain is lex-maximal, then by reversing the lexicographic order and invoking Lemma 9, we are done.

In order to obtain a contradiction, let $C=\sigma_{1} \triangleleft \cdots \triangleleft \sigma_{k}$ be the lex-maximal $\sigma$ - $\tau$-chain in $I_{n}$, and assume that it is not decreasing; say that $\lambda\left(\sigma_{1}, \sigma_{2}\right) \leq \lambda\left(\sigma_{2}, \sigma_{3}\right)$. By Lemma $8, \sigma_{1} \triangleleft \sigma_{2} \triangleleft \sigma_{3}$ is lexminimal among the $\sigma_{1}-\sigma_{3}$-chains in $I_{n}$. Hence, $\sigma_{1} \triangleleft \sigma_{2}^{\prime} \triangleleft \sigma_{3} \triangleleft \cdots \triangleleft \sigma_{k}$, where $\sigma_{1} \triangleleft \sigma_{2}^{\prime} \triangleleft \sigma_{3}$ is the decreasing $\sigma_{1}-\sigma_{3}$-chain, is lex-larger than $C$, which is a contradiction.

Is it possible to use the same idea to prove that every interval in $F_{n}^{A} \subset I_{n}$ is EL-shellable for some $A \neq\{0\}$ ? Unfortunately, the answer is no, since for all $A \neq\{0\}$ (except the trivial cases $A=\emptyset$ and $A=\{n\}$ ), it is possible to find $\sigma_{1}<\tau_{1}$ and $\sigma_{2}<\tau_{2}$ in $F_{n}^{A}$, such that the increasing $\sigma_{1}$ - $\tau_{1}$-chain and the decreasing $\sigma_{2}-\tau_{2}$-chain in $I_{n}$, are of length 2 and are not contained in $F_{n}^{A}$.

\section{Acknowledgements}

The author thanks Axel Hultman for helpful comments and fruitful discussions.

\section{References}

[1] A. Björner. Shellable and Cohen-Macaulay partially ordered sets. Trans. Amer. Math. Soc., 260: 159-183, 1980.

[2] A. Björner and F. Brenti. Combinatorics of Coxeter groups, volume 231 of Graduate Texts in Mathematics. Springer, New York, 2005.

[3] M. B. Can, Y. Cherniavsky, and T. Twelbeck. Lexicographic shellability of the Bruhat-Chevalley order on fixed-point-free involutions. Israel J. Math., to appear.

[4] P. H. Edelman. The Bruhat order of the symmetric group is lexicographically shellable. Proc. Amer. Math. Soc., 82:355-358, 1981. 
[5] S. Helgason. Differential geometry, Lie groups, and symmetric spaces, volume 80 of Pure and Applied Mathematics. Academic Press, New York, 1978.

[6] A. Hultman. Fixed points of involutive automorphisms of the Bruhat order. Adv. Math., 195:283296, 2005.

[7] A. Hultman. The combinatorics of twisted involutions in Coxeter groups. Trans. Amer. Math. Soc., 359:2787-2798, 2007.

[8] A. Hultman. Twisted identities in Coxeter groups. J. Algebraic Combin., 28:313-332, 2008.

[9] F. Incitti. The Bruhat order on the involutions of the symmetric group. J. Algebraic Combin., 20: 243-261, 2004.

[10] R. W. Richardson and T. A. Springer. The Bruhat order on symmetric varieties. Geom. Dedicata, 35:389-436, 1990.

[11] R. W. Richardson and T. A. Springer. Complements to: The Bruhat order on symmetric varieties. Geom. Dedicata, 49:231-238, 1994.

[12] R. P. Stanley. Enumerative combinatorics. Vol. 1, volume 49 of Cambridge Studies in Advanced Mathematics. Cambridge University Press, Cambridge, 1997. 
